Document downloaded from:

http://hdl.handle.net/10251/105533

This paper must be cited as:

Santiago-Portillo, A.; Navalón Oltra, S.; Concepción Heydorn, P.; Alvaro Rodríguez, MM.; García Gómez, H. (2017). Influence of Terephthalic Acid Substituents on the Catalytic Activity of MIL-101(Cr) in Three Lewis Acid Catalyzed Reactions. ChemCatChem. 9(13):2506-2511. doi:10.1002/cctc.201700236

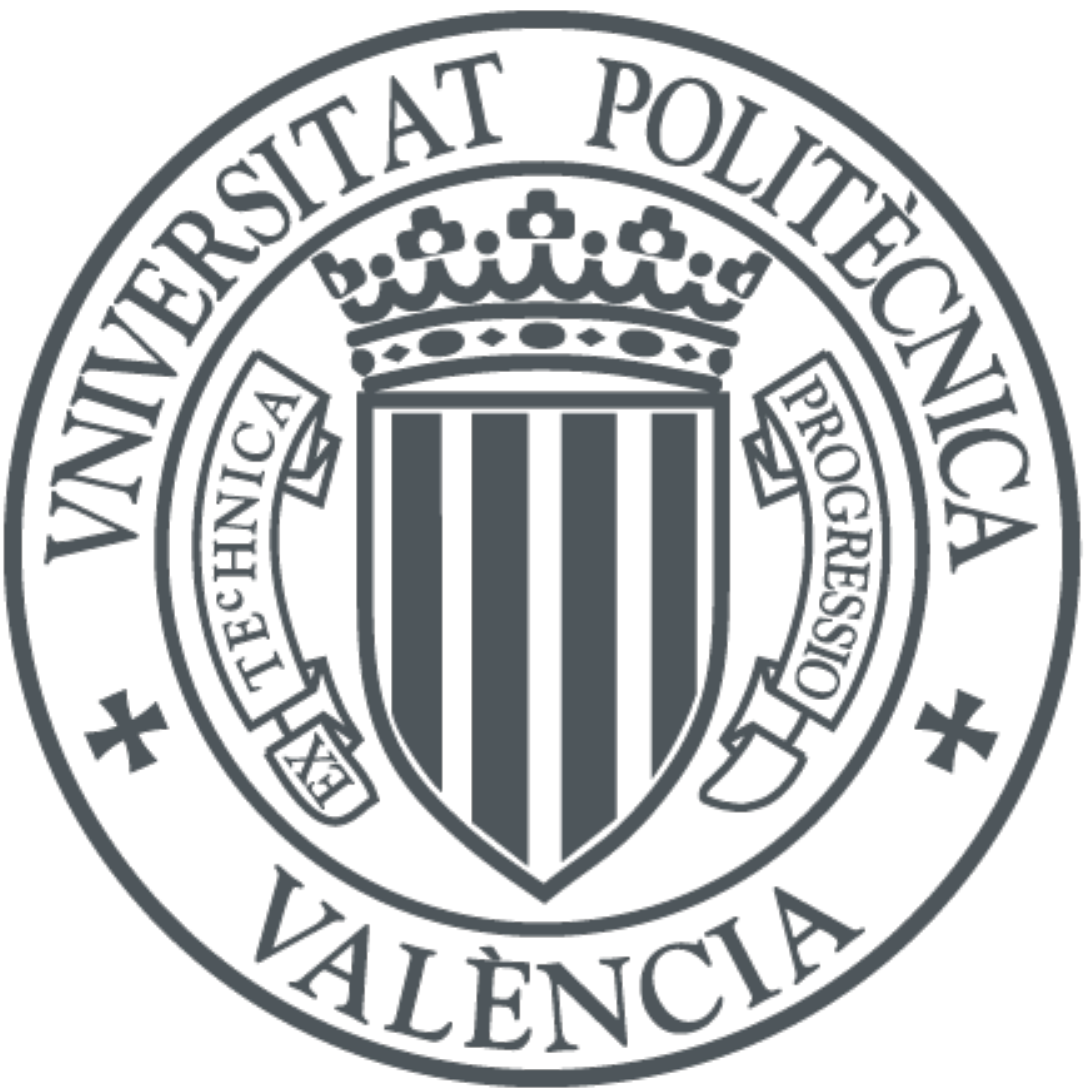

The final publication is available at

https://doi.org/10.1002/cctc.201700236

Copyright John Wiley \& Sons

Additional Information 


\title{
Influence of terephthalic acid substituents on the catalytic activity of MIL-101(Cr) for three Lewis acid catalyzed reactions.
}

\author{
Andrea Santiago-Portillo, ${ }^{[a]}$ Sergio Navalón, ${ }^{*[a]}$ Patricia Concepción, ${ }^{[b]}$ Mercedes Álvaro, ${ }^{[a]}$ and \\ Hermenegildo García*[a][b][c]
}

\begin{abstract}
Six isostructural MIL-101(Cr)-X (X: $\mathrm{H}, \mathrm{NO}_{2}, \mathrm{SO}_{3} \mathrm{H}, \mathrm{Cl}, \mathrm{CH}_{3}$ and $\mathrm{NH}_{2}$ ) have been prepared directly by reaction of $\mathrm{Cr}$ (III) salts and the corresponding terephthalic acid or by post-synthetic treatments of preformed MIL-101(Cr), following reported procedures. The materials have been characterized by XRD (crystallinity and coincident diffraction pattern), isothermal N2 adsorption (specific surface area ranging from 2740 for MIL-101(Cr)-H to $1250 \mathrm{~m}^{2} / \mathrm{g}$ for MIL-101(Cr)-Cl), Thermogravimetry (thermal stability up to $400^{\circ} \mathrm{C}$ ) and IR spectroscopy (detection of the corresponding substituents) that were all in agreement with the reported date for these materials. The series of MIL-101(Cr) were tested as heterogeneous catalyst for epoxide ring opening by methanol, benzaldehyde acetalization by methanol and Prins coupling, observing a clear influence on the substituent that in general follows a linear relationship with the Hammett $\sigma_{\mathrm{m}}$ constant of the substituent, the catalytic activity increases as the electron withdrawing ability of the substituents increases. Up to three orders of magnitude enhance by the present of $\mathrm{NO}_{2}$ substituent has been found for some of these reactions. The present study illustrates the versatility that MOFs offer as heterogeneous catalyst allowing the design of actives sites of adequate properties tuned for each reaction.
\end{abstract}

\section{Introduction}

Due to the large percentage of transition metal ions having frequently coordinative exchangeable position, their large porosity and accessibility of the intracrystalline voids, metal organic frameworks (MOFs) have become amount the most widely studied heterogeneous catalyst for the liquid phase reactions. ${ }^{[1-8]}$ While actives sites can also be located at the linkers or hosted on the empty spaces of MOFs, ${ }^{\left[{ }^{[]}\right.}$the most frequent case in the use of MOFs as heterogeneous catalyst is when metal nodes act as Lewis acid sites. ${ }^{[2,10-14]}$ In a pioneer study de Vos and coworkers have shown for UiO-66 that the catalytic activity of the material can be tuned and further

[a] Andrea Santiago-Portillo, Dr.S.Navalón, Dr Patricia Concepción, Prof.M.Álvaro Prof. H.García

Departamento de Química and Instituto de Tecnología Química CSIC-UPV, Universidad Politécnica de Valencia, Consejo Superior de Investigaciones Científicas, Av. de los Naranjos s/n, 46022 Valencia, Spain.

E-mail: hgarcia@qim.upv.es

[b] Center of Excellence for Advanced Materials Research, King Abdulaziz University, Jeddah, Saudi Arabia

Supporting information for this article is given via a link at the end of the document. enhanced by substitution in the aromatic ring of terephthalate linker. ${ }^{[10]}$ It was demonstrated that the electron donor or acceptor ability of substituents influences through inductive effects the electron density of the metal nodes and in this way the activity as Lewis acid centers can be altered. ${ }^{[10]}$ Considering the interest of MOFs as catalysts, it is necessary to expand this initial study to other MOFs particularly that have exhibited large stability and activity, to determine in which extent and for which reaction types this methodology based on the use of substituted aromatic linkers can be useful in catalysis. ${ }^{[2]}$

In this context, in the present work, we have selected MIL$101(\mathrm{Cr})$ and studied the influence of the presence of terephthalate substituents on three different types of Lewis acid catalyzed organic reactions. MIL-101 is constituted by trimeric $\mathrm{Cr}_{3}-\mu_{3} \mathrm{O}$ building units acting as nodal centers that are connected to six terephthalate linkers. ${ }^{[15-18]}$ Each $\mathrm{Cr}^{3+}$ ion is octahedrally coordinated by four terephthalate linkers, the $\mu_{3} \mathrm{O}$ atom and a sixth position that is occupied by fluoride ions in two of the three $\mathrm{Cr}$ atoms and by a solvent molecule in the third one. ${ }^{[15-18]}$ Figure 1 illustrates the structure of MIL-101(Cr), the nodes and linkers defining supertetrahedral units that arrange forming two types of cavities of 1.6 and $1.2 \mathrm{~nm}$ dimension, ${ }^{[18]}$ the smallest ones accessible through pentagonal windows $(5.5 \mathrm{~A})$ and the largest $(8.6 \mathrm{~A})$ ones through a hexagonal openings. ${ }^{[18]}$
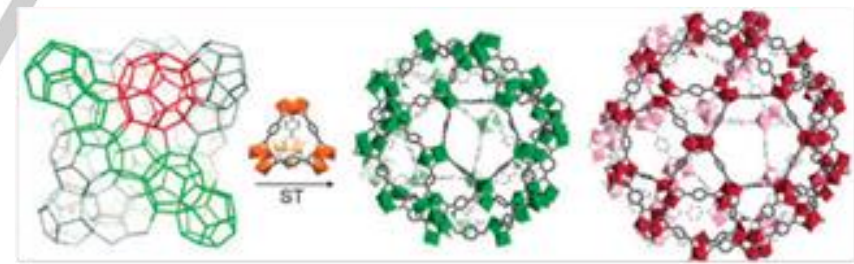

Figure 1. Schematic 3D representation of the MIL-101 structure made of smaller (green) and larger (red) cages connected through super tetrahedra (ST). The illustration shows in the left the ensemble of the two types of cages and in the right isolated cages of each type of cavity. Reprinted with permission from ref. ${ }^{[18]}$

MIL-101 has a very large surface area and pore volume with reported values of $2,700 \mathrm{~m}^{2} / \mathrm{g}$ and $2.1 \mathrm{~cm}^{3} / \mathrm{g}$, respectively. ${ }^{[16,18]}$ One of the most important features of MIL-101 is the reliability of its preparation procedure and the high crystallinity of the samples obtained. ${ }^{[16,18]}$ This situation contrasts with the initial case for which the influence of substituents on catalysis was reported, namely, UiO-66. ${ }^{[19,20]}$ It is known that UiO-66 can present in its structure a large proportion of defects, depending on the synthetic protocol used has been frequently reported. The presence of defects with metal ions having unsaturated positions would, certainly, overlay with the influence of 
substituents on the aromatic ring when determining the catalytic activity of the materials, thus, making uncertain the exact influence of substituents on the catalytic activity. ${ }^{[20]}$ In contrast, by selecting MIL-101 with low structural defects a more accurate confirmation of the importance of the inductive effects played by the substituent tuning the acid strength of the metal centers can be obtained. ${ }^{[16,21]}$ In addition, MIL-101(Cr) also enjoys a high structural, thermal and chemical stability that makes this material among the most robust and stable MOFs, a prerequisite necessary for its use as heterogeneous catalyst. ${ }^{[3,14,22-24]}$

\section{Results and Discussion}

\section{Catalyst characterization}

In the present study we have prepared six MIL-101(Cr)-X (X: H, $\mathrm{NO}_{2}, \mathrm{NH}_{2}, \mathrm{Cl}, \mathrm{SO}_{3} \mathrm{H}, \mathrm{CH}_{3}$ ) having different substituents on the terephthalate linker. Scheme S1 summarizes the preparation of each of the samples under study, while the experimental section contains the details, amounts of reagents, temperatures and times of the hydrothermal synthesis used in the preparation of the six MIL-101(Cr)-X. Four of them, including the parent MIL$101(\mathrm{Cr})-\mathrm{H}$, were obtained directly by reaction of chromium salts with the corresponding terephthalate under hydrothermal condition as previously reported. ${ }^{[16,25-27]}$ The MIL-101(Cr) $-\mathrm{SO}_{3} \mathrm{H}$ was obtained by reaction of $\mathrm{MIL}-101(\mathrm{Cr})-\mathrm{H}$ with $\mathrm{HSO}_{3} \mathrm{Cl},{ }^{[27]}$ while MIL-101(Cr)- $\mathrm{NH}_{2}$ was prepared from MIL-101(Cr)- $\mathrm{NO}_{2}$ chemical reduction by of the nitro groups with $\mathrm{SnCl}_{2}$ in acid media. ${ }^{[25]}$ The series of MIL-101(Cr)-X used in the present study have been already reported in the literature and in our case characterization data agrees with those already reported for these samples. ${ }^{[16,25-}$ 27] Specifically, PXRD diffraction of the samples exhibits the same pattern indicating that all of them are isoestructural (Figure 2). The broad X-ray diffraction peaks observed for the MIL$101(\mathrm{Cr})-\mathrm{X}$ materials are probably due to their small crystal sizes ranging from 100 to $400 \mathrm{~nm}$, as revealed by SEM images (Figure S1). In the particular case of MIL-101(Cr)- $\mathrm{CH}_{3}$, two types of crystals of submicrometric and micrometric sizes were also observed in SEM.

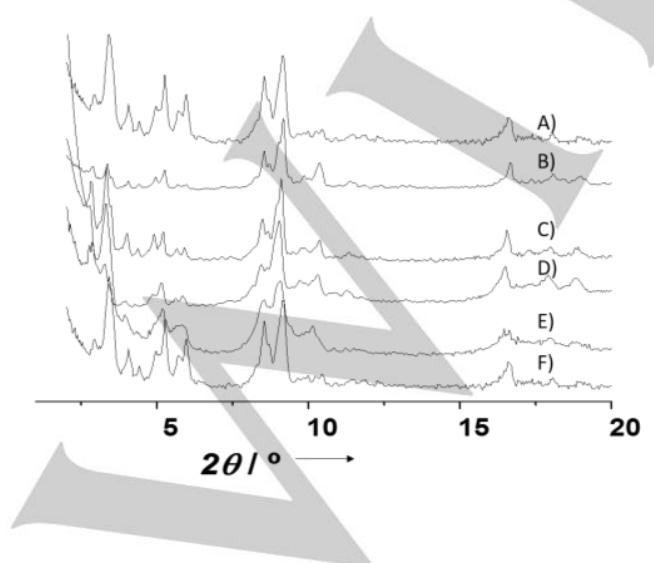

Figure 2 XRD patterns of MIL-101(Cr) $-\mathrm{NO}_{2}$ (a), MIL-101(Cr)-SO ${ }_{3} \mathrm{H}$ (b), MIL$101(\mathrm{Cr})-\mathrm{Cl}$ (c), MIL-101(Cr)-H (d), MIL-101(Cr)-CH (e) and MIL-101(Cr)-NH (f).

Isothermal nitrogen adsorption measurements (Figures S2-S7) revealed that $\mathrm{MIL}-101(\mathrm{Cr})-\mathrm{H}$ is the one that exhibits the largest specific surface area $\left(2,750 \mathrm{~m}^{2} \mathrm{~g}^{-1}\right)$ and pore volume $\left(2.2 \mathrm{~cm}^{3} \mathrm{~g}\right.$ 1) (Table S1). The rest of the samples have lower specific surface area and porosity volume ranging between 1,950 and $1,300 \mathrm{~m}^{2} \mathrm{~g}^{-1}$ and from 2.0 to $1.1 \mathrm{~cm}^{3} \mathrm{~g}^{-1}$ (Table S1). ${ }^{[24,26]}$ The decrease in surface area and porosity when substituents are present on the aromatic ring was already noticed in the literature and attributed to the intracrystalline space required to accommodate the substituents. ${ }^{[26]}$

The presence of substituents and their nature can be determined by infrared spectroscopy ${ }^{[26]}$ wherein the specific vibration peaks expected for each substituent were recorded. Figures S8-S14 show the relevant region of the IR spectra where the characteristic vibration bands corresponding to each substituent have been indicated. IR spectra are particularly relevant to follow post-synthetic modification of MIL-101(Cr)-H and $\mathrm{MIL}-101(\mathrm{Cr})-\mathrm{NO}_{2}$ leading to the formation of MIL-101(Cr)$\mathrm{SO}_{3} \mathrm{H}$ and $\mathrm{MIL}-101(\mathrm{Cr})-\mathrm{NH}_{2}$, respectively, by monitoring the disappearance of the $\mathrm{NO}_{2}$ bands $\left(1500-1380 \mathrm{~cm}^{-1}\right)$ and the appearance of the characteristic vibrations of $\mathrm{SO}_{3} \mathrm{H}\left(1,160 \mathrm{~cm}^{-1}\right)$ and $\mathrm{NH}_{2}\left(3,450\right.$ and $\left.1,625 \mathrm{~cm}^{-1}\right)$.

Thermogravimetric analyses in air of MIL-101(Cr) materials show their stability up to about $300 \stackrel{\circ}{\circ}$ (Figures S15-S20). In general, a good relationship between the theoretical and the experimental chromium content for all the MIL-101(Cr) materials prepared in the present work was determined (Table S1). ${ }^{[26]}$ In the case of MIL-101(Cr)-Cl, the slightly higher metal content experimentally observed $(\sim 1.65 \%)$ respect to the theoretical value may indicate the presence of some amount of chromium oxide inside the cavities of the material. In addition, the FT-IR spectrum of MIL-101(Cr)-Cl (Figures S8 and S12) presents a weak band at around $1,710 \mathrm{~cm}^{-1}$ that could be ascribed to the carbonyl stretching band of the non-coordinated free terephthalic acid. Thus, in MIL-101(Cr)-Cl either the presence of chromium oxide or the presence of free terephthalic acid inside the cavities, may be the reason for their lower than expected BET surface area and pore volume. In addition, these impurities could be responsible for the lower intensity of the $\mathrm{X}$-ray diffraction peaks at low $2 \theta$ angles characteristic of the pore symmetry in the MOF.

\section{Catalytic activity}

As commented earlier the purpose of this study was to assess the influence of substituents on the terephthalate linker on the catalytic activity of the MIL-101(Cr)-X for three different acid catalyzed reactions (Scheme 1). Aimed at this purpose, the ring opening of epoxide by methanol (Figure 3), benzaldehyde acetalization by methanol (Figure 4) and the Prins coupling of $\beta$ pinene and formaldehyde (Figure 5) and the reactions carried out in the presence of the set of MIL-101(Cr)-X as heterogeneous catalysts. 
Ring opening of epoxides

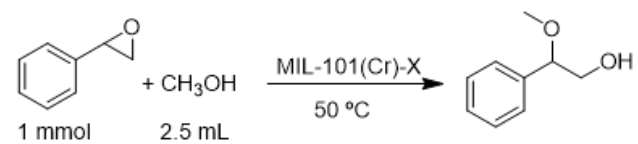

Acetalization of aldehydes<smiles>COC(CO)c1cccc(CC(C)C(C)C)c1</smiles>

Prins reaction

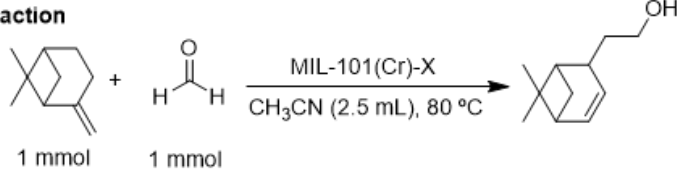

Scheme 1. Three acid-catalyzed reactions that were studied using the series of MIL-101(Cr) solids as heterogeneous catalysts. The temporal profiles of substrate conversion depending on the substituent on the terephthalate linker are presented in Figures 3-5. As it can be seen there, the three reaction types follow exactly the same relative activity order for the set of catalysts, MIL$101(\mathrm{Cr})-\mathrm{NO}_{2}$ being far more active than the rest of catalyst. Figures 3-5 include also the corresponding plot of the relative initial reaction rate in logarithmic scale versus the $\sigma_{\mathrm{m}}$ Hammett constant of the substituent.
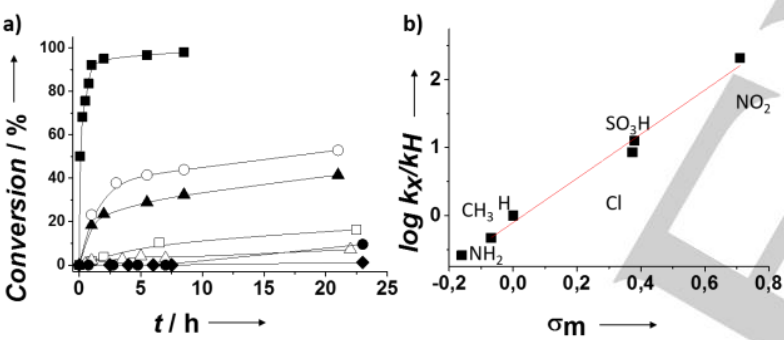

Figure 3. a) Time-conversion plot for the ring opening of styrene oxide by methanol using each MIL-101(Cr)-X as catalyst. Legend: MIL-101(Cr)-H ( $\square$ ), $\mathrm{MIL}-101(\mathrm{Cr})-\mathrm{NO}_{2}(\boldsymbol{\square}), \quad \mathrm{MIL}-101(\mathrm{Cr})-\mathrm{SO}_{3} \mathrm{H}$ (O), MIL-101(Cr)-Cl ( $\left.\mathbf{\Delta}\right)$, MIL$101(\mathrm{Cr})-\mathrm{CH}_{3}(\Delta), \mathrm{MIL}-101(\mathrm{Cr})-\mathrm{NH}_{2}(\bullet)$ and blank control in the absence of catalyst $(\diamond)$. Reaction conditions: Catalyst $(0.04 \mathrm{mmol}$ of $\mathrm{Cr}$ ), styrene oxide (1 $\mathrm{mmol})$, methanol $(2.5 \mathrm{~mL}), 50^{\circ} \mathrm{C}$. b) Plot of the logarithm of the relative initial reaction rate vs the $\sigma_{\mathrm{m}}$ Hammett constant of each substituent on the terephthalate linker.

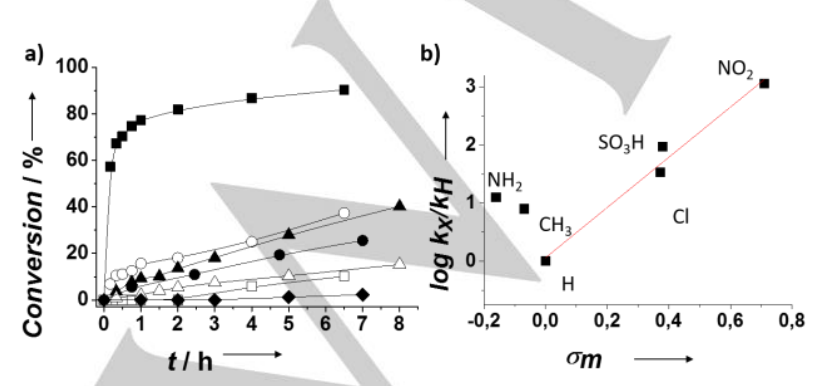

Figure 4. a) Time-conversion plot for the benzaldehyde acetalization using each MIL-101(Cr)-X as catalyst. Legend: MIL-101(Cr)-H ( $\square)$, MIL-101(Cr)-NO
(ש), MIL-101(Cr)-SO ${ }_{3} \mathrm{H}(0), \mathrm{MIL}-101(\mathrm{Cr})-\mathrm{Cl}(\boldsymbol{\Delta}), \mathrm{MIL}-101(\mathrm{Cr})-\mathrm{CH}_{3}(\Delta), \mathrm{MIL}$ $101(\mathrm{Cr})-\mathrm{NH}_{2}(\bullet)$ and blank control in the absence of catalyst $(\bullet)$. Reaction conditions: Catalyst $(0.04 \mathrm{mmol}$ of $\mathrm{Cr})$, benzaldehyde $(1 \mathrm{mmol})$, methanol $(3$ $\mathrm{mL}$ ), $30^{\circ} \mathrm{C}$. b) Plot of the logarithm of the relative initial reaction rate vs the $\sigma_{\mathrm{m}}$ Hammett constant of each substituent on the terephthalate linker.

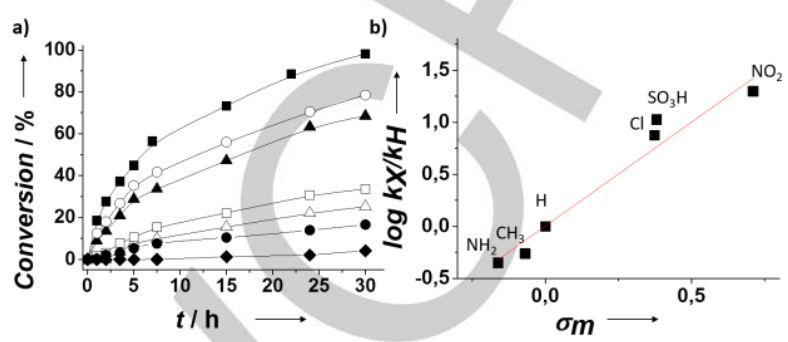

Figure 5. a) Time-conversion plot for the Prins coupling of $\beta$-pinene using each MIL-101(Cr)-X as catalyst. Legend: MIL-101(Cr)-H ( $\square)$, MIL-101(Cr)-NO $(\boldsymbol{\square}), \mathrm{MIL}-101(\mathrm{Cr})-\mathrm{SO}_{3} \mathrm{H}(\mathrm{O}), \mathrm{MIL}-101(\mathrm{Cr})-\mathrm{Cl}(\boldsymbol{\Delta}), \mathrm{MIL}-101(\mathrm{Cr})-\mathrm{CH}_{3}(\Delta), \mathrm{MIL}-$ $101(\mathrm{Cr})-\mathrm{NH}_{2}(\bullet)$ and blank control in the absence of catalyst $(\bullet)$. Reaction conditions: Catalyst $(0.08 \mathrm{mmol}$ of $\mathrm{Cr}), \beta$-pinene $(1 \mathrm{mmol})$, formaldehyde $(1$ $\mathrm{mmol})$, acetonitrile $(2.5 \mathrm{~mL}), 80^{\circ} \mathrm{C}$. b) Plot of the logarithm of the relative initial reaction rate vs the $\sigma \mathrm{m}$ Hammett constant of each substituent on the terephthalate linker.

In the precedent in the literature, ${ }^{[10]}$ the $\sigma_{\text {meta }}$ Hammett constant of the substituent on the aromatic ring was selected as the representative parameter to correlate the relative initial reaction rates due to the better linearity respect to $\sigma_{\text {ortho. This was also }}$ the parameter selected in the present study. As it can be seen in these Figures, epoxide ring opening (Figure 3) and Prins coupling (Figure 5) follow a relatively good linear relationship between catalytic activity to the material and $\sigma_{\mathrm{m}}$ Hammett constant, while in the case of benzaldehyde acetalization, MIL$101(\mathrm{Cr})-\mathrm{X}$ having electron donor substituents $\left(\mathrm{CH}_{3}\right.$ and $\left.\mathrm{NH}_{2}\right)$ have higher catalytic activity than expected according to $\sigma_{\mathrm{m}}$ values (Figure 4). In the case of epoxide ring opening the initial reaction rate changes four orders of magnitude from $\mathrm{NH}_{2}$ (the less active catalyst; $0.00058 \mathrm{mmol} \mathrm{h}^{-1}$ ) to the $\mathrm{NO}_{2}$ substituent $\left(5.49 \mathrm{mmol} \mathrm{h}^{-1}\right)$ and the catalytic activity of the $\mathrm{NO}_{2}$ substituted catalyst increases more than 300 times respect to the parent MIL-101(Cr) $\left(0,018 \mathrm{mmol} \mathrm{h}^{-1}\right)$. Although similar trend is also observed for the Prins reaction, in this case, the influence of the substituent is not so remarkable, the initial relative reaction rate increasing from the $\mathrm{NH}_{2}\left(0.00078 \mathrm{mmol} \mathrm{h}^{-1}\right)$ to the $\mathrm{NO}_{2}(0.166$ $\mathrm{mmol} \mathrm{h}{ }^{-1}$ ) functionalized material by 200 times, the $\mathrm{NO}_{2}$ catalyst being about 8 times more active than the parent MIL-101(Cr) $\left(0.021 \mathrm{mmol} \mathrm{h}^{-1}\right)$.

To put these values in context, it has to be indicated that in the case of substituted UiO-66-X previously reported the enhanced of catalytic activity for the cyclization of citronellal was 56 times.

For the case of benzaldehyde acetalization, also a remarkable three orders of magnitude enhanced of the catalytic activity by introduction of $\mathrm{NO}_{2}$ group $\left(3.105 \mathrm{mmol} \mathrm{h}^{-1}\right)$ versus the parent MIL-101(Cr) $\left(0.0113 \mathrm{mmol} \mathrm{h}^{-1}\right)$ was observed, although as commented above the $\mathrm{NH}_{2}$ substituted material was significantly more efficient (about two orders of magnitude) than expected by considering exclusively the $\sigma_{\mathrm{m}}$ Hammett constant. Since in the 
previous two reactions a good linearity has been observed, it is unlikely that this deviation of the linearity could be due to some defects on the MOF structure or some other structural factor. ${ }^{[10,}$ ${ }^{14]}$ It is suggested that the higher than expected activity of MIL$101(\mathrm{Cr})-\mathrm{NH}_{2}$ should reflect the operation of an alternative reaction mechanism in which besides metal centers with tunable electron density by the terephthalate linker, the amino groups play active role, probably interacting with the carbonyl group of benzaldehyde and opening new pathways for acetalization, likely involving an imine. ${ }^{[14,28]}$ It is also possible that other factors like different adsorption ability could contribute to the observed deviation of the linearity.

In order to correlate the observed catalytic activity with the Lewis acidity of the sample, $\mathrm{CO}$ adsorption monitored by IR spectroscopy has been used for Lewis acid titration (Figure 6). ${ }^{[29]}$ Figure 6 shows the IR spectra of CO adsorbed on MIL-101(Cr)$\mathrm{NO}_{2}, \mathrm{MIL}-101(\mathrm{Cr})-\mathrm{NH}_{2}$ and $\mathrm{MIL}-101(\mathrm{Cr})-\mathrm{H}$ catalysts, observing a shift in the value of the $v(\mathrm{CO})$ frequency that appears at 2198 $\mathrm{cm}^{-1}$ for the most active MIL-101(Cr)- $\mathrm{NO}_{2}$ sample, $2194 \mathrm{~cm}^{-1}$ for the parent MIL-101 (Cr)-H sample, and $2191 \mathrm{~cm}^{-1}$ for the less active MIL-101(Cr)- $\mathrm{NH}_{2}$ sample. The observed $\mathrm{CO}$ frequencies are due to the interaction of $\mathrm{CO}$ with $\mathrm{Cr}^{3+},{ }^{[30]}$ where a shift to higher frequencies should correspond to a higher Lewis acidity of the $\mathrm{Cr}^{3+}$ site. Accordingly, a quantitative scale of Lewis acidity can be determined for the samples MIL-101(Cr)-X, following the order MIL-101(Cr)-NO $\mathrm{NO}_{2}>$ MIL-101(Cr)-H> MIL-101(Cr)-NH which correlate with the catalytic activity of the samples.

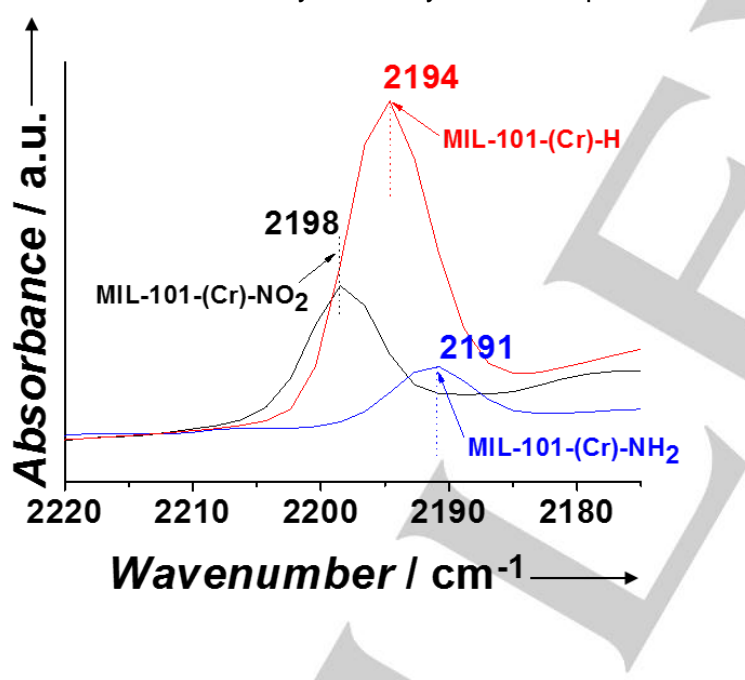

Figure 6. FTIR spectra of $\mathrm{CO}$ adsorption at $6 \mathrm{mbar}$ and $-176{ }^{\circ} \mathrm{C}$ on MIL-101 (Cr)-NO $\mathrm{NO}_{2}$ MIL-101 (Cr)-NH $\mathrm{NH}_{2}$ and MIL-101 (Cr)-H samples.

The higher catalytic activity of MIL-101(Cr)- $\mathrm{NO}_{2}$ respect to MIL$101(\mathrm{Cr})-\mathrm{NH}_{2}$ is mainly attributed to electronegative inductive effects, therefore, increasing the Lewis acidity of the solid material as the inductive effect of the substituent in the aromatic ring increases. As commented before, the MIL-101(Cr)- $\mathrm{NH}_{2}$ material was obtained by chemical reduction of preformed MIL$101(\mathrm{Cr})-\mathrm{NO}_{2}$. This chemical reaction does not apparently alters the morphology of the MOF particles and SEM images reveal that the shape and particle size distribution are almost coincident for MIL-101(Cr)- $\mathrm{NO}_{2}$ and $\mathrm{MIL}-101(\mathrm{Cr})-\mathrm{NH}_{2}$ (Figure $\mathrm{S} 1)$. This characterization data reinforces that the increase of catalytic activity of MIL-101(Cr) materials tested in the present work for three acid catalyzed reactions should be attributed to the higher Lewis acidity induced by the presence of electron withdrawing groups.

The absence of metal leaching and the occurrence of truly heterogeneous reaction mechanisms were demonstrated in the cases of MIL-101(Cr)- $\mathrm{NO}_{2}$ by analysis of the liquid phase after the reactions and by hot filtration tests (Figures S21-S23). Chemical analysis shows that the amount of $\mathrm{Cr}$ at the end of the reactions is lower than $0.5 \%$ of the total chromium present in the initial catalyst. Control experiments under the same conditions using the amount of chromium determined by chemical analysis show no activity for the reactions under consideration under the conditions employed in the presence study (Figure $7 \mathrm{a}$ ). In the case of the hot filtration test, the solid catalyst was removed at the reaction temperature, once the reaction has been initiated and conversion reached about $50 \%$. It can be seen in Figures S21-S23 that the reactions stop once the solid catalyst is removed from the reaction.

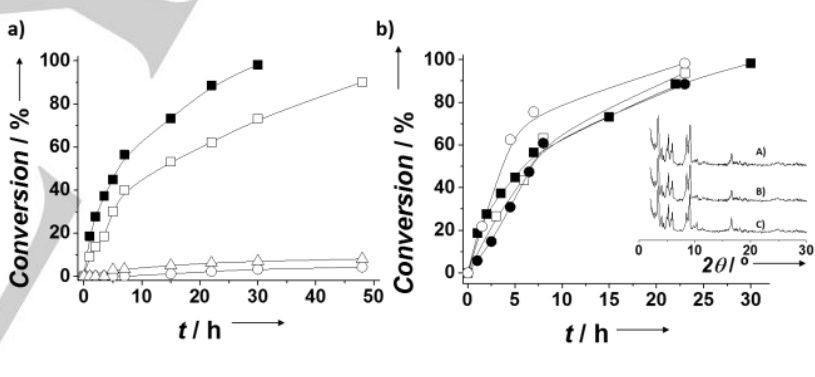

Figure 7. Time-conversion plot for the Prins coupling of $\beta$-pinene using MIL$101(\mathrm{Cr})-\mathrm{NO}_{2}$ and Chromium acetate as catalyst. Legend: $\mathrm{MIL}-101(\mathrm{Cr})-\mathrm{NO}_{2}(\mathbf{\square})$, chromium acetate in the same amount of MIL-101(Cr) $-\mathrm{NO}_{2}(\square)$, chromium acetate in the same amount of the leaching measured in MIL-101(Cr)- $\mathrm{NO}_{2}(\mathrm{O})$ and chromium acetate in double amount than the leaching occurring in MIL$101(\mathrm{Cr})-\mathrm{NO}_{2}(\Delta)$. Reaction conditions: Catalyst $(0.08 \mathrm{mmol}$ of $\mathrm{Cr}), \beta$-pinene (1 $\mathrm{mmol})$, formaldehyde $(1 \mathrm{mmol})$, acetonitrile $(2.5 \mathrm{~mL}), 80 \stackrel{\circ}{\circ}$. The inset of pane b) shows the XRD of fresh MIL-101(Cr) $-\mathrm{NO}_{2}(\mathrm{~A})$, three times used MIL$101(\mathrm{Cr})-\mathrm{NO}_{2}(\mathrm{~B})$ and four times used MIL-101(Cr)-NO $2(\mathrm{C})$.

Also in the case of $\mathrm{MIL}-101(\mathrm{Cr})-\mathrm{NO}_{2}$ reusability was confirmed by performing a series of consecutive runs using the same sample for Prins reaction (Figure $7 b$ ). At the end of each reaction cycle, the catalyst was separated by filtration, washed twice with hot ethanol and dried before the next use. As it can be seen in Figure $7 b$ the catalytic activity as well as the crystallinity of the solid material is maintained upon reuse.

Finally, the catalytic activity of MIL-101(Cr)- $\mathrm{NO}_{2}$ was compared with the activity of chromium acetate as homogeneous catalyst under the same conditions. The results are presented in Figure 
7. As it can be seen there, chromium acetate is also active to promote the Prins coupling, but MIL-101(Cr) $-\mathrm{NO}_{2}$ exhibits higher initial rate and also higher conversion and higher final conversion at $48 \mathrm{~h}$ than the soluble chromium salt, this showing the advantage of introducing an adequate substituent to enhance the catalytic activity of active sites. When comparing homogeneous and heterogeneous catalyst, it is a general observation that immobilization of the active sites on a solid results in lower activity due to diffusion restrictions of substrates and reagents to reach the sites that are less accessible than when they are dissolved in a liquid phase. Although this could be the case when comparing the catalytic activity of chromium acetate in $\mathrm{CH}_{3} \mathrm{CN}$ solution with MIL-101(Cr)- $\mathrm{H}$, the possibility to introduce an electron withdrawing substituent on the terephthalate linker renders the solid catalyst far more active than the homogeneous $\mathrm{Cr}$ (III) salt.

\section{Conclusions}

In the present work it has been shown that, in agreement with previous reports, the catalytic activity of MOFs as solids Lewis acids can be increased by the presence of electron withdrawing substituents on the organic linker that by inductive effects can increase the electronic deficiency on the nodal metal ions acting as catalytic centers. In the present case, up to three orders of magnitude enhancement has been determined when comparing the activity of the amino and nitro substituted MIL-101(Cr)-X. With respect to the studies reported in the literature, the present study has the advantage of being based on MIL-101 that is known to be one of the most crystalline MOFs structure is less prone to the presence of structural defects, thus, providing evidence in support of the influence of the substituent on firm ground. Two of the reactions studied epoxide ring opening and Prins coupling, exhibit a high linearity between the relative initial reaction rates in logarithm scale and the $\sigma_{\mathrm{m}}$ Hammett constant, suggesting that no other factors besides acidity are influencing in the process.

\section{Experimental Section}

Materials. All the reagents and solvents used in this work were of analytical or HPLC grade and supplied by Sigma-Aldrich.

Catalyst preparation. Isoestructural MIL-101(Cr)-X (X: $\mathrm{H}, \mathrm{NO}_{2}, \mathrm{SO}_{3} \mathrm{H}$, $\mathrm{Cl}, \mathrm{CH}_{3}$ and $\mathrm{NH}_{2}$ ) materials were prepared following already reported procedures. ${ }^{[16,25-27]}$ Briefly, the corresponding substituted terephtalic acid (1.5 mmol) and $\mathrm{Cr}\left(\mathrm{NO}_{3}\right)_{3} \cdot 9 \mathrm{H}_{2} \mathrm{O}$ (for the synthesis of $\left.\mathrm{MIL}-101(\mathrm{Cr})-\mathrm{H}\right)$ or $\mathrm{CrCl}_{3}$ (for the synthesis of $\mathrm{MIL}-101(\mathrm{Cr})-\mathrm{X}\left(\mathrm{X}: \mathrm{NO}_{2}, \mathrm{Cl}, \mathrm{CH}_{3}\right)(1 \mathrm{mmol})$ were introduced into a Teflon autoclave containing demineralized water (8 mL). Subsequently, in the case of MIL-101(Cr)-H, HF $(10 \mu \mathrm{L})$ was added. Then, the autoclave was heated at the corresponding temperature for a specific period of time (see Table 1 for synthesis conditions for each MIL-101(Cr)-X). Once the autoclave was cooled at room temperature, the formed precipitate was washed several times with dimethylformamide (DMF) at $120{ }^{\circ} \mathrm{C}$ and, then, with ethanol at $80{ }^{\circ} \mathrm{C}$ MIL-101(Cr)- $\mathrm{NH}_{2}$ material was prepared by post-synthetic reduction of MIL-101(Cr) $-\mathrm{NO}_{2}$ with $\mathrm{SnCl}_{2} \cdot \mathrm{H}_{2} \mathrm{O}$ according to reported procedures. ${ }^{[25]}$ In the case of $\mathrm{MIL}-101(\mathrm{Cr})-\mathrm{SO}_{3} \mathrm{H}$, the material was obtained by postsynthetic sulfonation of MIL-101(Cr)-H with chlorosulfonic acid $\left(\mathrm{HSO}_{3} \mathrm{Cl}\right)$ as previously reported in the literature. ${ }^{[27]}$

Table 1. Temperature and time employed in the preparation of MIL-101(Cr)-X materials.

\begin{tabular}{lll} 
materials. & \\
\hline & Temperature $(\stackrel{\circ}{ } \mathrm{C})$ & Time $(\mathrm{h})$ \\
\hline $\mathrm{MIL101}(\mathrm{Cr})-\mathrm{H}$ & 200 & 8 \\
$\mathrm{MIL101}(\mathrm{Cr})-\mathrm{NO}_{2}$ & 180 & 120 \\
$\mathrm{MIL101}(\mathrm{Cr})-\mathrm{Cl}^{\prime}$ & 180 & 96 \\
$\mathrm{MIL101}(\mathrm{Cr})-\mathrm{CH}_{3}$ & 180 & 120
\end{tabular}

Catalyst characterization. Powder X-ray diffraction (PXRD) patterns of MIL-101(Cr)-X materials were recorded on a Philips XPert diffractometer equipped with a graphite monochromator (40 kV and $45 \mathrm{~mA}$ ) employing Ni filtered CuKa radiation. ATR-FTIR spectra of MIL-101(Cr)-X materials were collected using a Bruker Tensor27 instrument after heating the samples were heated in an oven ( $100 \stackrel{\circ}{\mathrm{C}}$ for $16 \mathrm{~h})$ to remove physisorbed water. CO-FTIR spectra were recorded in a Nexus 8700 FTIR spectrophotometer using an IR cell allowing in situ treatments at controlled temperature, from $-176{ }^{\circ} \mathrm{C}$ to $500{ }^{\circ} \mathrm{C}$, and connected to a high vacuum system with gas dosing facility. For $\mathrm{CO}$ adsorption measurements the samples were pressed into self-supported wafers and treated in vacuum $\left(10^{-6} \mathrm{mbar}\right)$ at $150{ }^{\circ} \mathrm{C}$ for $2 \mathrm{~h}$. After activation, the wafers were cooled down to $-176{ }^{\circ} \mathrm{C}$ under dynamic vacuum followed by CO dosing at increasing pressure (0.4-6 mbar). IR spectra were collected after each dosage. All IR spectra corresponding to $\mathrm{CO}$ adsorption measurement have been normalized to the weight of the IR wafer. $\mathrm{N}_{2}$ 
adsorption isotherms at $77 \mathrm{~K}$ were recorded using a Micromeritics ASAP 2010 device. Termogravimetric analyses were performed on a TGA/SDTA851e METTLER TOLEDO station. Scanning electron microscopy (SEM) images were collected using a Zeiss instrument.

Catalytic experiments. Briefly, the required amount of MIL-101(Cr)-X (X: $\mathrm{H}, \mathrm{NO}_{2}, \mathrm{NH}_{2}, \mathrm{Cl}, \mathrm{CH}_{3}$ ) employed as catalyst $(0.04 \mathrm{mmol}$ of $\mathrm{Cr}$ for ring opening of epoxides and acetalization or 0.08 for the Prins reaction) was introduced into a two-necked round-bottom flask $(25 \mathrm{~mL})$. Then, the MIL$101(\mathrm{Cr})$ materials were pretreated at $150 \stackrel{\circ}{ } \mathrm{C}$ under vacuum for $16 \mathrm{~h}$ to remove solvent molecules and generate coordinatively unsaturated sites at the metal clusters. Subsequently, the system was cool down to the required reaction temperature. In the case of the ring opening epoxides, styrene oxide $(1 \mathrm{mmol})$ dissolved in methanol $(2.5 \mathrm{~mL})$ was added to the round-bottom flask containing the activated catalyst previously stabilized at $50{ }^{\circ} \mathrm{C}$. In the acetalization of aldehydes, benzaldehyde $(1 \mathrm{mmol})$ dissolved in methanol $(3 \mathrm{~mL})$ was introduced at $30{ }^{\circ} \mathrm{C}$ in the roundbottom flask containing the activated catalyst. In the Prins reaction, $\beta$ pinene $(1 \mathrm{mmol})$ and formaldehyde $(1 \mathrm{mmol})$ dissolved in acetonitrile $(2.5$ $\mathrm{mL}$ ) were added to the round-bottom flask containing the activated catalyst that was equilibrated at $80 \stackrel{\circ}{ } \mathrm{C}$. The reactions were magnetically stirred and reaction aliquots sampled at the corresponding reaction times.

Catalyst reusability, for the most active MIL-101(Cr)- $\mathrm{NO}_{2}$ sample was studied for the Prins reaction. In these experiments, the solid catalyst was recovered at the end of the reaction by filtration (Nylon filter, $0.2 \mu \mathrm{m}$ ) Then, the catalyst was transferred to a round-bottom flask $(50 \mathrm{~mL})$ and submitted under magnetic stirring to ethanol washings $(20 \mathrm{~mL})$ at $80 \stackrel{\circ}{\circ}$ for $2 \mathrm{~h}$. This procedure was repeated two more times. The solid was recovered by filtration (Nylon filter, $0.2 \mu \mathrm{m}$ ) and dried in an oven at 100 ${ }^{\circ} \mathrm{C}$ for $24 \mathrm{~h}$. The catalyst was activated at $150^{\circ} \mathrm{C}$, under vacuum for $16 \mathrm{~h}$ before a new catalytic cycle.

Product analysis. Previously filtered reaction aliquots were diluted in methanol or acetonitrile solution containing a known amount of nitrobenzene as external standard in the case of the ring opening epoxide and acetalization reactions or Prins reaction, respectively. The aliquots were immediately analyzed by chromatography using a flame ionization detector. Quantification was carried out by using calibration curves of authentic samples against nitrobenzene.

Leaching experiments. At the end of the Prins reaction, the most active MIL-101(Cr)-NO2 sample was removed by filtration (Nylon filter, $0.2 \mu \mathrm{m}$ ). Then, the organic phase was stirred with $\mathrm{HNO}_{3}$ aqueous solution ( $3 \mathrm{M}$ ), and the system heated at $80{ }^{\circ} \mathrm{C}$ for $24 \mathrm{~h}$ to extract $\mathrm{Cr}^{3+}$ ions. The presence of chromium in the aqueous phase was analyzed by chemical analysis using an ICP-AES instrument.

\section{Acknowledgements}

Financial support by the Spanish Ministry of Economy and Competitiveness (Severo Ochoa and CTQ2015-69153 and CTQ2014-53292-R and Generalitat Valenciana (Prometeo 2013/14) and is grateful acknowledged. SN thanks financial support by the Fundación Ramón Areces (XVIII Concurso Nacional para la Adjudicación de Ayudas a la Investigación en Ciencias de la Vida y de la Materia, 2016).

Keywords: Heterogeneous catalysis - Lewis acid catalysis • metal-organic frameworks $\bullet$ effect of ligand substituent $\bullet$ tuning of catalytic activity

[1] A. H. Chughtai, N. Ahmad, H. A. Younus, A. Laypkov, F. Verpoort, Chem. Soc. Rev. 2015, 44, 6804-49.

[2] A. Corma, H. Garcia, F. X. Llabrés i Xamena, Chem. Rev. 2010, 110, 4606-55.

[3] A. Dhakshinamoorthy, Opanasenko, M., Čejka, J., Garcia, H., Catal. Sci. Technol. 2013, 3, 2509-40.

[4] A. Dhakshinamoorthy, M. Alvaro, H. Garcia, Catal. Sci. Technol. 2011, 1, 85667.

[5] H. Furukawa, Cordova, K.E., O'Keeffe, M., Yaghi, O.M., Science 2013, 341, 1230444.

[6] J. Gascon, A. Corma, F. Kapteijn, F. X. Llabrés i Xamena, ACS Catal. 2014, 4, 361-78.

[7] O. A. Kholdeeva, Catal. Today 2016, 278, 22-9.

[8] M. Yoon, R. Srirambalaji, K. Kim, Chem. Rev. 2012, 112, 1196-231. 
[9] Y.-X. Zhou, Y.-Z. Chen, Y. Hu, G. Huang, S.-H. Yu, H.-L. Jiang, Chem. Eur. J. 2014, 20, 14976-80.

[10] F. Vermoortele, M. Vandichel, B. V. de Voorde, R. Ameloot, M. Waroquier, V. Van Speybroeck, D. E. De Vos, Angew. Chem. Int. Ed. 2012, 51, 4887-90.

[11] B. Li, K. Leng, Y. Zhang, J. J. Dynes, J. Wang, Y. Hu, D. Ma, Z. Shi, L. Zhu, D. Zhang, Y. Sun, M. Chrzanowski, S. Ma, J. Am. Chem. Soc. 2015, 137, 4243-8.

[12] A. Herbst, A. Khutia, C. Janiak, Inorg. Chem. 2014, 53, 7319-33.

[13] C. K. Brozek, M. Dincă, J. Am. Chem. Soc. 2013, 135, 12886-91.

[14] A. Santiago-Portillo, J. F. Blandez, S. Navalón, M. Álvaro, H. García, Catal. Sci. Technol. (doi: 10.1039/c6cy02577c) 2017.

[15] Y. F. Chen, R. Babarao, S. I. Sandler, J. W. Jiang, Langmuir 2010, 26, 8743-50.

[16] G. Ferey, C. Mellot-Draznieks, C. Serre, F. Millange, J. Dutour, S. Surble, I. Margiolaki, Science 2005, 309, 2040-2.

[17] N. V. Maksimchuk, O. V. Zalomaeva, I. Y. Skobelev, K. A. Kovalenko, V. P. Fedin, O. A. Kholdeeva, Proc. R. Soc. A 2012, 468, 2017-34.

[18] A. Demessence, P. Patricia Horcajada, C. Christian Serre, C. C. Boissière, D. Grosso, C. Sanchez, G. Férey, Chem. Commun. 2009, 7149-51.

[19] H. Wu, Y. S. Chua, V. Krungleviciute, M. Tyagi, P. Chen, T. Yildirim, W. Zhou, J. Am. Chem. Soc. 2013, 135, 10525-32.

[20] Z. Fang, B. Bueken, D. E. De Vos, R. A. Fischer, Ang. Chem. Int. Ed 2015, 54, 7234-54.

[21] D.-Y. Hong, Y. K. Hwang, C. Serre, G. Férey, J.-S. Chang, Adv. Funct. Mater. 2009, 19, 1537-52.

[22] A. Dhakshinamoorthy, H. Garcia, Chem. Soc. Rev. 2012, 41, 5262-84.

[23] A. Gómez-Paricio, A. Santiago-Portillo, S. Navalón, P. Concepción, M. Alvaro, H. Garcia, Green Chem. 2016, 508-15.
[24] A. Santiago-Portillo, S. Navalon, F. Cirujano, F. Llabrés i Xamena, M. Alvaro, H. Garcia, ACS Catal. 2015, 5, 3216-24.

[25] S. Bernt, V. Guillerm, C. Serre, N. Stock, Chem. Commun. 2011, 47, 2838-40.

[26] M. Lammert, S. Bernt, F. Vermoortele, D. E. De Vos, N. Stock, Inorg. Chem. 2013, 52, 8521-8.

[27] B. Li, Y. Zhang, D. Ma, L. Li, G. Li, G. Li, Z. Shi, S. Feng, Chem. Commun. 2012, 48, 6151-3.

[28] M. Hartmann, M. Fischer, Micropor. Mesopor. Mater. 2012, 164, 38-43.

[29] M. I. Zaki, H. Knözinger, Spectrochim. Acta 1987, 43A, 1455- 9.

[30] K. Hadjiivanov, G. N. Vayssilov $A d v$. Catal. 2002, 47, 307-511. 
Entry for the Table of Contents (Please choose one layout)

Layout 1:

\section{FULL PAPER}

Electron withdrawing substituents on terephthalate ligand of MIL-101(Cr)-X enhance the catalytic activity of the material for Lewis acid catalyzed reactions

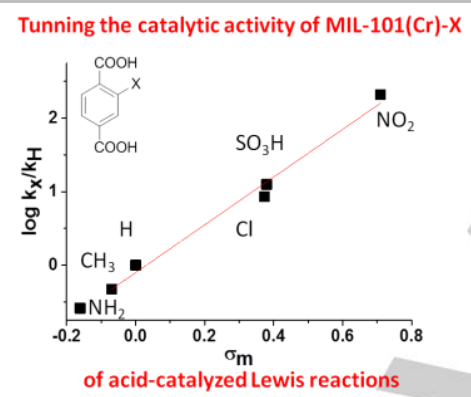

Andrea Santiago-Portillo, Sergio Navalón, Patricia Concepción, Mercedes Álvaro, Hermenegildo García*

Page No. - Page No.

Title 